\title{
L'ALIMENTATION DU POISSON-CHAT (ICTALURUS MELAS RAF.) DANS LE LAC DE CRETEIL
}

\author{
par Ph. BOËT1
}

\begin{abstract}
Le régime alimentaire de 276 poissons-chats (Ictalurus melas Rafinesque, 1818) capturés entre septembre 1977 et août 1978 est analysé par une approche classique de l'alimentation naturelle.

Le caractère omnivore de l'espèce est vérifié et les différences de comportement suivant la taille des individus sont très nettes. Les jeunes individus sont planctonophages; ils se nourrissent de Copépodes et de Cladocères. La nourriture des adultes est d'origine benthique, dominée par les larves de Chironomides. Les plus gros poissons-chats ont un comportement ichtyophage mais ils s'attaquent principalement aux Gardons (Rutilus rutilus L.) parasités par la ligulose.

L'étude des variations saisonnières de la composition du régime alimentaire confirme l'opportunisme des poissons-chats qui font preuve d'une grande facilité d'adaptation aux contraintes physiques et biotiques du milieu.
\end{abstract}

\section{Feeding of catfish (Ictalurus melas Raf.) in the lake of Créteil.}

The feeding of 276 catfishes (Ictalurus melas Rafinesque, 1818) captured between September 1977 and August 1978 was studied by the classical approach of natural food analysis.

The omnivorous character of this species was confirmed and differences between fish were clearly related to the size of the fish. Young fish feed on plankton : copepods and cladocerans. Adult fish feed on benthic animals especially chironomid larvae. The largest catfish are piscivores and chiefly attack roach (Rutilus rutilus L.) that are parasitized by ligulids.

The study of seasonal variations in diet confirms the opportunism of catfish and shows that they have the capacity to adapt to the physical and biotic constraints of the environment.

Ictalurus melas (Rafinesque, 1818) est originaire d'Amérique du Nord où le régime alimentaire des Ictaluridés a déjà fait l'objet de plusieurs travaux. Bailley et Harrisson (1948), Heard (1975), Walburg (1975) et Klarberg et Benson (1975) ont mis en évidence le caractère omnivore et opportuniste des différentes espèces étudiées : les Crustacés et les larves de Diptères sont la base de leur alimentation, les variations ontogéniques de comportement sont très prononcées et les jeunes individus se nourrissent principalement de zooplancton.

1. Laboratoire de Zoologie, Ecole Normale Supérieure, 46, rue d'Ulm, 75230 Paris cedex 05. 
Les études récentes de Repsys, Applegate et Hales (1976) sur le lac Poinsett (Sud Dakota) confirment l'opportunisme de I. melas qui tire sa nourriture d'une grande variété de sources dans les zones limnétique, littorale et benthique, et dont le régime alimentaire varie saison. nièrement selon la disponibilité des espèces proies. Cependant l'étude de ses préférences montre que c'est avant tout un poisson essentiellement planctonophage pendant tout son cycle vital, sélectionnant surtout les grandes espèces de Cladocères.

La présente note étudie le comportement alimentaire d'I. melas aans le lac de Créteil, ses variations selon la taille des individus et les saisons, par une approche classique de l'alimentation naturelle; elle s'intègre dans une étude écologique globale de ce milieu d'origine artificielle.

\section{1. - MILIEU ET METHODES}

\subsection{Le milieu d'étude}

Le lac de Créteil (Val-de-Marne) est établi sur d'anciennes gravières situées entre la Marne et la Seine. Sa superficie est de 35 ha, la profondeur moyenne de $4 \mathrm{~m}$, avec un maximum de $6 \mathrm{~m}$. C'est une cuvette fermée, alimentée uniquement par la nappe phréatique et les eaux de pluies. Le taux de renouvellement est faible, estimé à 1/250 (Chesterikoff, 1979). La minéralisation est très forte $\left(1,3\right.$ à $\left.1,6 \mathrm{~g} \mathrm{t}^{-1}\right)$ caractérisée par de fortes teneurs en sulfates $\left(600-700 \mathrm{mg} \mathrm{l}^{-1}\right)$ (Testard, à parâ̂tre).

La température varie de $2{ }^{\circ} \mathrm{C}$ en été, mais elle n'a jamais dépassé $21^{\circ} \mathrm{C}$ pendant l'été exceptionnellement frais de l'année 1978 (fig. 1).

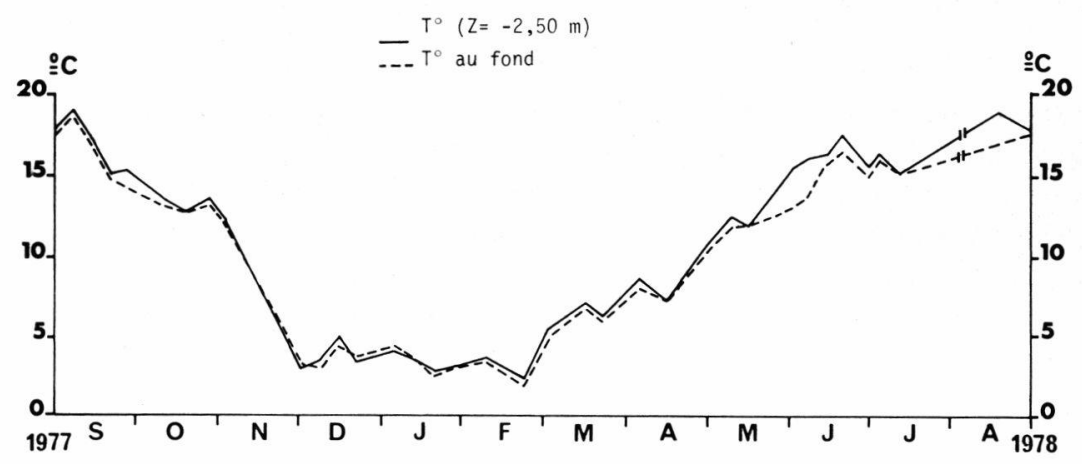

FIg. 1. - Variations saisonnières (sept. 1977 à sept. 1978) de la température de l'eau dans la partie profonde du lac. 
L'été, la tendance à la stratification est nette; le déficit en oxygène est permanent et très prononcé à partir de 2,50-3,00 m (fig. 2).

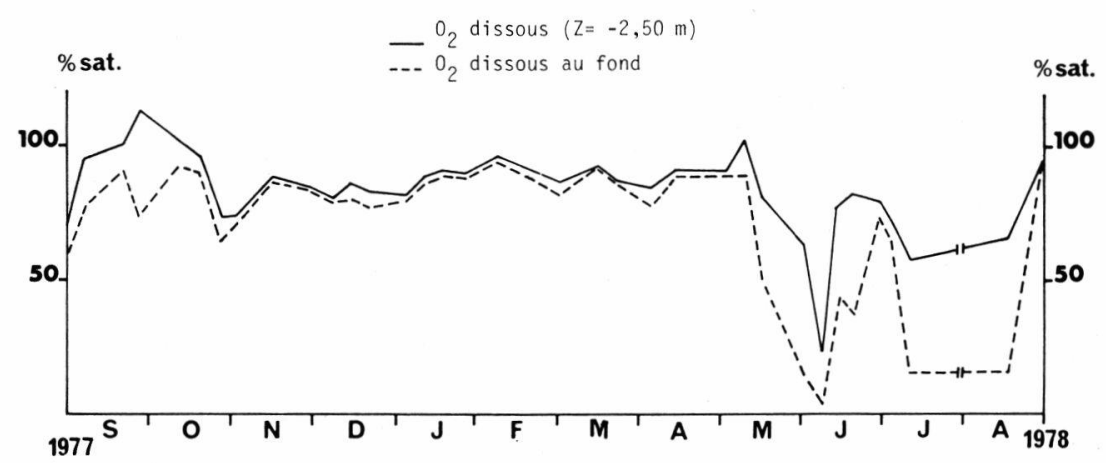

Fig. 2. - Variations saisonnières (sept. 1977 à sept. 1978) de l'oxygène dissous dans la partie profonde du lac.

Le milieu est potentiellement eutrophe, mais à faible biomasse phytoplanctonique (Chlorophycées et Diatomées) et à très faible transparence $(80 \mathrm{~cm}$ en moyenne au disque de Secchi).

La zone littorale est étroite, les rives étant très abruptes. On y trouve des Dreissena polymorpha Pall., surtout sur le talus, quelques Paludines (Vivipara sp.), des Physes (Physa sp.), des écrevisses (Orconectes limosus Raf.), quelques Aselles et une espèce de Trichoptères. Quelques Typhaies et Phragmitaies abritent une faune classique d'herbiers : des Cératopogonidés, au moins deux espèces d'Odonatés (1 Zygoptère, 1 Anisoptère), des Hémiptères (Corixidés parfois abondants et Népidés) et des Coléoptères.

La zone profonde s'étend sur plus de 30 ha. Le fond est vaseux dans sa plus grande étendue et limoneux à ses extrémités, généralement pauvre en matière organique (Chesterikoff, 1979 ; Guiral, à parâ̂tre). Le zoobenthos est composé d'une importante population de Chironomides dominés par $C$. plumosus $\mathrm{L}$. ( $90 \%$ de la biomasse totale) et d'Oligochètes (Tubificidés). On trouve aussi des écrevisses, quelques Ostracodes et des Chydoridés (C. sphaericus Müil.).

Dans la zone pélagique, le peuplement zooplanctonique est dominé par l'espèce Bosmina longirostris Mül., les Copépodes sont bien représentés, avec trois espèces de Cyclopides et deux espèces de Calanides ; Acanthocyclops robustus (Sars) domine largement. Six espèces principales de Rotifères ont été observées.

Le peuplement de poissons est bien diversifié : 11 espèces ont été dénombrées, parmi lesquelles la Brème (Abramis brama L.), le Gar- 
don (Rutilus rutilus L.), le Poisson-chat, la Perche (Perca fluviatilis L.) et le Sandre (Lucioperca lucioperca L.) se développent bien. L'espèce Poisson-chat est représentée par une population abondante avec des individus âgés de grande taille.

\section{2. - Echantillonnage}

Deux procédés ont été utilisés : les pièges passifs (nasses et filets maillants) et la pêche électrique.

Malgré la très forte conductivité de l'eau (1 500 à $1800 \mu \mathrm{S} \mathrm{cm}^{-1}$ ), la pêche à l'électricité a été largement employée pour réaliser des échantillonnages réguliers grâce à un chalut électrifié original. Avec des tensions de crête de 110 à 220 volts celui-ci s'est avéré très efficace dans la capture des poissons-chats en eaux profondes et peu sélectif par rapport aux tailles des individus (Gerdeaux et Jestin 1979). Ce chalut à perche, de petite taille $(80 \mathrm{~cm}$ d'ouverture), est trainé à $35 \mathrm{~m}$ derrière un bateau à une vitesse réduite de $3 \mathrm{~km} . \mathrm{h}^{-1}$ environ. Chaque trait dure cinq minutes afin de limiter la pêche à une zone précise. A la fin du trait, le chalut est remonté sans stopper l'avance du bateau, et le courant est coupé au dernier moment.

L'été, où le déficit en oxygène contraint les poissons-chats à remonier vers les zones littorale et superficielle, le chalut cesse d'être efficace en zone profonde. Des méthodes classiques de pêche (nasses et filets maillants) ont donc été employées, bien qu'elles n'aient pas une efficacité entièrement satisfaisante et qu'elles ne permettent pas de connaître l'heure exacte des captures. Les prélèvements étaient complétés par des pêches électriques à pied le long des berges. Pendant les trois premiers mois de l'étude (septembre, octobre et novembre) avant la mise au point du chalut, seules les techniques de captures passives avaient été utilisées.

Bien qu'il existe un doute quant à l'influence de l'électricité sur d'éventuelles régurgitations (Neveu 1978), le risque est moins imporiant qu'avec les filets maillants (Keast et Welsh 1968) ou les nasses.

Enfin les captures ont été pratiquées dans une même tranche horaire aussi réduite que possible, en milieu de journée, afin de limiter les biais éventuels dus à l'influence de l'heure de capture.

\subsection{Stockage et préservation}

Les poissons sont tués dès leur capture et une injection de formol (4\%) est immédiatement pratiquée dans le tube digestif pour éviter toute digestion et décomposition. L'appareil digestif entier est ensuite prélevé au laboratoire et stocké dans du formol (4\%) avant d'être 
analysé. Pour chaque individu, les données de base suivantes sont notées : date, heure et mode de capture, sexe, longueur totale et poids trais éviscéré.

\subsection{Analyse des contenus du tube digestif}

L'analyse porte sur l'ensemble des contenus stomacaux et intestinaux (Davis 1977). La méthode choisie, décrite par Hynes (1950), ne tient compte que de la fréquence d'observation et du dénombrement de chaque catégorie d'aliments. Les résultats sont exprimés en pourcentage du nombre total de poissons examinés (fréquences d'occurence) ou en nombre moyen d'organismes ingérés par poisson.

Dans certains cas où le dénombrement est rendu difficile par l'état dégradé des fragments d'organismes rencontrés, des évaluations des quantités ingérées ont été faites à partir des débris présents afin de tirer une information plus riche que la seule notion de présence/ absence. Cinq classes d'abondance ont été distinguées :

$$
\begin{aligned}
& \text { classe } 1: \text { absence } \\
& \text { classe } 2: n<25 \\
& \text { classe } 3: 25 \leqslant n<50 \\
& \text { classe } 4: 50 \leqslant n<100 \\
& \text { classe } 5: n \geqslant 100
\end{aligned}
$$

Pour certains calculs, la classe 5 est subdivisée en sous-classes d'amplitude 50 (ex. : 5a: 150, 5b: 200, ...)

L'état trophique, degré de remplissage du tube digestif, est estimé de la manière suivante :

$$
\begin{aligned}
& \text { dlasse } 1 \text { : estomac et intestin vides } \\
& \text { classe } 2 \text { : estomac vide } \\
& \text { classe } 3: \text { estomac peu rempli } \\
& \text { classe } 4: \text { estomac moyennement rempli } \\
& \text { classe } 5 \text { : estomac plein. }
\end{aligned}
$$

\section{2. - RESULTATS}

Des prélèvements mensuels de septembre 1977 à août 1978 ont permis l'étude de 276 poissons-chats. Toutes les captures ont été faites en fin de matinée entre $10 \mathrm{~h}$ et $14 \mathrm{~h}$. L'effectif de chaque échantillon varie de 7 à 45 individus (25 en moyenne) et la taille des poissons est comprise entre 4 et $31 \mathrm{~cm}$.

L'utilisation des nasses et des filets maillants introduit un biais important dans la structure en taille et l'équilibre des échantillons mensuels, car ces techniques ont une efficacité variable et sont très 
sélectives (fig. 3). Tous les poissons capturés dans les nasses, par exemple, ont entre $10 \mathrm{~cm}$ et $20 \mathrm{~cm}$ ide longueur.

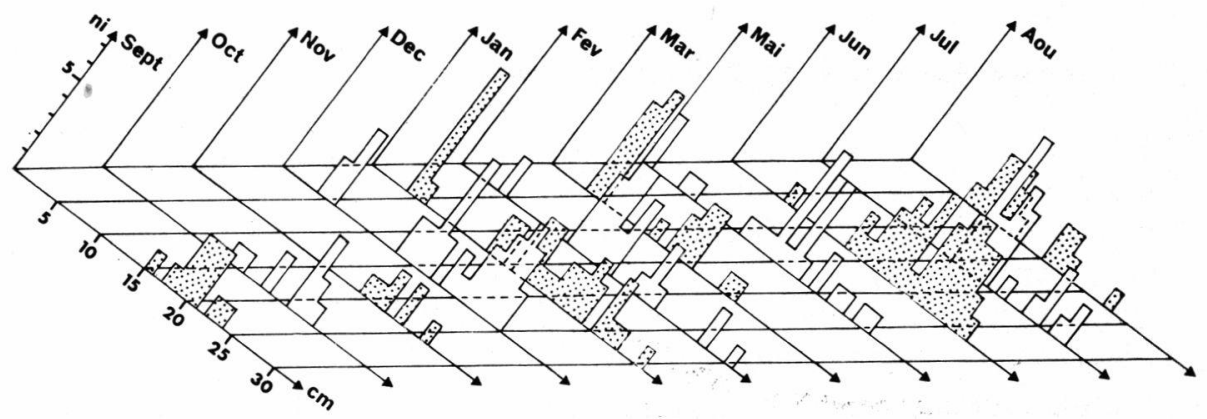

Fig. 3. - Histogramme des tailles des poissons-chats de chaque échantillon mensuel. (Il n'y a pas eu de prélèvement au mois d'avril).

Globalement, l'image de la population est sensiblement conforme aux modèles simples de la dynamique des populations. La cohorte des individus de 1'année $(4-10 \mathrm{~cm})$ est facile à distinguer, mais très sous-estimée en nombre. Parmi les individus plus âgés (10 $\mathrm{cm}$ et plus) apparaissent les cohortes correspondant à un, deux ans et plus.

Le taux de féminité, de 110 femelles pour 206 individus, soit 0,53, n'est pas significativement différent de $1 / 2$ (le sexe de 70 poissons n'a pas été déterminé).

\subsection{Rythme saisonnier d'activité alimentaire}

On peut apprécier le rythme saisonnier d'activité d'un poisson en comparant le pourcentage d'estomacs vides observés dans la population à diverses époques de l'année (Mc Cormack 1970 ; Davis 1977). La figure 4 résume d'une part l'évolution du pourcentage de tubes digestifs (estomacs et intestins) vides (fig. 4 a) et d'autre part celle du seull pourcentage d'estomacs vides (fig. $4 \mathrm{~b}$ ). Ces deux courbes sont très semblables excepté durant l'été où l'augmentation de la vitesse du transit gastrique se traduit par un fort pourcentage d'estomacs vides. Dans les deux cas, les variations observées apparaissent bien influencées par les facteurs physiques de l'environnement $\left(t^{\circ}\right.$ et $\mathrm{O}_{2}$, fig. 1 et 2).

La valeur des résultats des trois premiers mois (septembre, octobre et novembre) est discutable car les échantillons sont peu représentatifs. On voit bien cependant que l'activité des poissons-chats diminue à partir de l'automne jusqu'à être minimale en hiver, aux mois de janvier et février qui correspondent aux températures les 

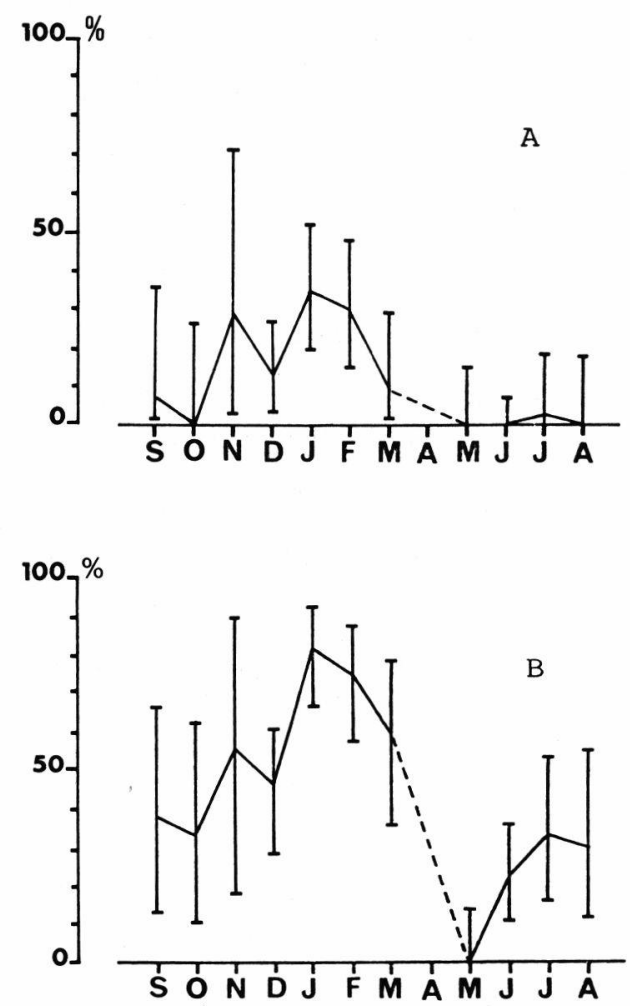

Frc. 4. - Rythme saisonnier d'activité alimentaire. \% d'estomacs et d'intestins vides (fig. $4 \mathrm{a}$ ); \% d'estomacs vides (fig. 4 b) et limite de l'intervalle de confiances à $95 \%$.

plus basses (autour de $3{ }^{\circ} \mathrm{C}$ en moyenne et un minimum de $1,9^{\circ} \mathrm{C}$ ). On peut remarquer en particulier qu'un léger réchauffement des eaux en décembre $\left(4,5^{\circ} \mathrm{C}\right)$ se traduit bien par un regain d'activité.

Au printemps, l'activité alimentaire augmente et devient maximale au début de l'été. Elle diminue à nouveau pendant la période estivale d'anoxie du fond.

\subsection{Analyse du régime alimentaire}

L'identification des aliments est possible dans l'estomac, mais elle est beaucoup plus délicate dans l'intestin où, le plus souvent, seuls les éléments chitineux des insectes sont identifiables et dénombrables (capsules céphaliques de Chironomides par exemple).

Pour certains groupes comme les éléments du zooplancton ou les 
Oligochètes, les calculs du nombre moyen d'organismes par poisson ont été faits en prenant la limite supérieure de la classe d'abondance. Les résultats ne donnent donc qu'un ordre de grandeur.

Cette méthode du dénombrement n'a pas pu être utilisée pour toutes les catégories d'aliments (algues filamenteuses et diatomées, frai...) ou n'avait pas de sens (débris végétaux, organismes d'origine exogène...).

\subsubsection{RÉGIME GLOBAL DU POISSON-CHAT}

Le tableau 1 fait apparaître une très grande variété de proies qui confirme le caractère omnivore de l'espèce. La nourriture de base est

\begin{tabular}{|c|c|c|c|c|}
\hline & $\begin{array}{c}\text { Fréquences } \\
\text { d'observation }\end{array}$ & $\begin{array}{l}\text { Fréquences } \\
\text { d'occurence }\end{array}$ & $\begin{array}{l}\text { Nbre total } \\
\text { d'individus }\end{array}$ & $\begin{array}{l}\text { Nbre moyen } \\
\text { par poisson }\end{array}$ \\
\hline Diptères & 205 & 74,2 & 14874 & 53,9 \\
\hline Larves de Chironomes & 204 & 73,2 & 13132 & 47,5 \\
\hline Nymphes de Chironomes & 91 & 32,9 & 1709 & 6,1 \\
\hline Autres Diptères & 15 & 5,4 & 33 & 0,1 \\
\hline Zooplancton & 139 & 50,3 & 19279 & 69,8 \\
\hline Copépodes & 116 & 42,0 & 12049 & 43,6 \\
\hline Cladocères & 80 & 28,9 & 3554 & 12,8 \\
\hline 0stracodes & 16 & 5,7 & 117 & 0,4 \\
\hline Rotifères & 2 & 0,7 & 5 & 0,01 \\
\hline 01 igochètes & 107 & 38,7 & 461 & 8,9 \\
\hline Poissons & 40 & 14,4 & 40 & 0,14 \\
\hline Gardons & 27 & 9,7 & 27 & 0,09 \\
\hline Autres & 13 & 4,7 & 13 & 0,04 \\
\hline Ecrevisses & 14 & 5,0 & 17 & 0,06 \\
\hline Divers & 33 & 11,9 & - & - \\
\hline Algues Fil. et Diat. & 9 & 3,2 & - & - \\
\hline Colèoptēres & 3 & 1,08 & 5 & 0,01 \\
\hline Hémiptères & 6 & 2,17 & 9 & 0,03 \\
\hline Mollusques & 4 & 1,44 & 13 & 0,04 \\
\hline Aselles & 7 & 2,53 & 53 & 0,19 \\
\hline Frai & 5 & 1,81 & - & - \\
\hline Débris végétaux & 7 & 2,5 & - & - \\
\hline $\begin{array}{l}\text { Terrestre (araignées, fourmis, } \\
\text { asticots,graines, etc...) }\end{array}$ & 9 & 3,2 & - & - \\
\hline
\end{tabular}

Tableau I. - Consommation globale des poissons-chats étudiés. 
toutefois composée principalement de Diptères (surtout des larves de Chironomides), de zooplancton (largement dominé par les Copépodes), d'Oligochètes et de poissons (deux fois plus de Gardons que d'autres espèces). Ces quatre groupes ont les plus fortes fréquences, voisines respectivement de $75 \%, 50 \%, 40 \%$ et $15 \%$. La faible occurence des autres catégories d'organismes laisse penser qu'il s'agit de nourritures occasionnelles.

Ces premières constatations s'accordent bien avec les ressources offertes par le milieu.

\subsubsection{COMPOSITION DU RÉGIME ALIMENTAIRE EN FONCTION DE LA TAILLE}

En répartissant la population en classes de tailles dont les limites ont été arbitrairement choisies de 5 en $5 \mathrm{~cm}$, les tableaux 2 et 3 font nettement apparaître les variations de comportement alimentaire du poisson chat selon son âge.

Les plus jeunes individus se nourrissent de quelques larves de Chironomides et surtout de zooplancton où les Copépodes et les Cladocères de grande taille sont leurs proies favorites.

En quantité, les rations ingérées augmentent ensuite rapidement avec l'âge. La fréquence des Chironomides (larves et nymphes) et des Oligochètes devient plus importante.

$\mathrm{Au}$ cours de leur croissance, le spectre alimentaire des poissonschats s'élargit progressivement. Leur nourriture de base se compose alors de Diptères (larves et nymphes de Chironomides), de zooplancton (Copépodes) et d'Oligochètes.

Il semble qu'à une consommation accrue d'Oligochètes et de larves de Chironomides corresponde une augmentation de la consommation de Cladocères. Ce phénomène est très certainement dû à la présence de cadavres de Bosmines, en très grand nombre dans les sédiments du lac, qui auront été ingérés accidentellement en même temps que le zoobenthos.

A partir de $15 \mathrm{~cm}$, les poissons-chats s'attaquent aux autres poissons, ainsi qu'aux écrevisses. La consommation importante de Gardons s'explique par leur vulnérabilité : ils sont en effet parasités par la Ligule (Ligula intestinalis, Cestodes Pseudophyllidés) dans de fortes proportions et les poissons malades ont une nage difficile.

La diversité du régime alimentaire des plus vieux poissons-chats (25 $\mathrm{cm}$ et plus) diminue. Ils cessent de se nourrir de zooplancton et ont des tendances ichtyophages très nettes. La prédation sur les écrevisses est également importante en quantité et il est possible d'observer 2 voire 3 individus dans l'estomac d'un gros poisson-chat. 


\begin{tabular}{|c|c|c|c|c|c|c|}
\hline Effectif de classe & 17 & 23 & 46 & 91 & 81 & 25 \\
\hline Diptẽres & 23,5 & 69,5 & 80,4 & 90,1 & 70,3 & 32,0 \\
\hline Larves de Chironomes & 23,5 & 69,5 & 80,4 & 90,1 & 70,3 & 28,0 \\
\hline Nymphes de Chironomes & - & 39,1 & 30,4 & 48,3 & 23,4 & 16,0 \\
\hline Autres Diptères & - & - & 2,1 & 5,4 & 7,4 & 4,0 \\
\hline Zooplancton & 82,3 & 100,0 & 50,0 & 64,8 & 23,4 & 4,0 \\
\hline Copépodes & 70,5 & 95,6 & 43,4 & 54,9 & 13,5 & 4,0 \\
\hline Cladocères & 52,9 & 56,5 & 13,0 & 24,1 & 12,3 & - \\
\hline Ostracodes & 23,5 & 17,3 & 6,5 & 2,1 & 2,4 & - \\
\hline Rotifères & 5,8 & 4,3 & - & - & - & - \\
\hline 01 igochètes & 5,8 & 17,3 & 19,5 & 57,1 & 39,5 & 38,8 \\
\hline Poissons & - & - & 4,3 & 13,1 & 24,6 & 33,3 \\
\hline Gardons & - & - & 4,3 & 9,8 & 16,0 & 16,6 \\
\hline Autres & - & - & - & 3,2 & 8,6 & 16,6 \\
\hline Ecrevisses & - & - & - & 2,1 & 14,8 & 1,2 \\
\hline Divers & - & 4,3 & 6,5 & 14,2 & 16,0 & 8,0 \\
\hline Algues Fil. et Diat. & - & - & 2,1 & 4,3 & 4,9 & - \\
\hline Coléoptères & - & - & 4,3 & - & 1,2 & - \\
\hline Hēmiptères & - & - & 4,3 & 1,0 & 3,7 & - \\
\hline Mollusques & - & - & - & 1,0 & 2,4 & 5,5 \\
\hline Aselles & - & - & 2,1 & 1,0 & 6,1 & - \\
\hline Frai & - & - & 4,3 & 3,2 & 1,2 & - \\
\hline Débris végētaux & - & - & - & 3,2 & 4,9 & - \\
\hline $\begin{array}{l}\text { Terrestre (araignēes,fourmis } \\
\text { asticots,graines,etc...) }\end{array}$ & - & 4,3 & - & 3,2 & 4,9 & 4,0 \\
\hline
\end{tabular}

TABLEAU II. - Régime alimentaire des poissons-chats des différentes classes de tailles (méthode de l'occurence; les résultats sont exprimés en pourcentages). 


\begin{tabular}{|c|c|c|c|c|c|c|}
\hline Classes de tailles &.$<5 \mathrm{~cm}$ & $5-10 \mathrm{~cm}$ & $0-15 \mathrm{~cm}$ & $5-20 \mathrm{~cm}$ & $0-25 \mathrm{~cm}$ & $25 \mathrm{~cm}$ \\
\hline Effectif de classes & 17 & 23 & 46 & 91 & 81 & 25 \\
\hline Diptères & 0,41 & 11,9 & 30,4 & 86,2 & 54,6 & 31,1 \\
\hline Larves de Chironomes & - & 11,9 & 30,4 & 86,1 & 54,4 & 30,7 \\
\hline Nymphes de Chironomes & - & 0,9 & 6,6 & 9,6 & 4,3 & 8,1 \\
\hline Autres Diptères & - & - & 0,02 & 0,07 & 0,19 & 0,33 \\
\hline Zooplancton & 21,1 & 195,0 & 60,0 & 72,5 & 15,5 & 8,3 \\
\hline Copépodes & 13,4 & 126,2 & 44,2 & 67,6 & 7,0 & 8,3 \\
\hline Cladocères & 6,17 & 67,3 & 15,7 & 4,7 & 8,4 & - \\
\hline Ostracodes & 1,35 & 1,3 & 0,45 & 0,14 & 0,08 & - \\
\hline Rotifères & 0,17 & 0,08 & - & - & - & - \\
\hline 01 igochètes & 0,05 & 1,0 & 1,8 & 16,0 & 9,8 & 2,6 \\
\hline Poissons & - & - & 0,04 & 0,13 & 0,24 & 0,33 \\
\hline Gardons & - & - & 0,04 & 0,09 & 0,16 & 0,16 \\
\hline Autres & - & - & - & 0,03 & 0,08 & 0,16 \\
\hline Ecrevisses & - & - & - & 0,02 & 0,14 & 0,11 \\
\hline \multicolumn{7}{|l|}{ Divers } \\
\hline Coléoptères & - & - & 0,04 & - & 0,03 & - \\
\hline Hémiptères & - & - & 0,04 & 0,01 & 0,07 & - \\
\hline Mollusques & - & - & - & 0,03 & 0,11 & 0,05 \\
\hline Aselles & - & - & 0,08 & 0,06 & 0,53 & - \\
\hline
\end{tabular}

TABlEAU III. - Régime alimentaire des poissons-chats des différentes classes de tailles (méthode du dénombrement: les résultats sont exprimés en nombre moyen d'organismes par poissons).

\subsubsection{VARIATIONS SAISONNIÈRES DE LA COMPOSITION DU RÉGIME ALIMEN- TAIRE}

Les variations saisonnières sont appréhendées en faisant une analyse de la composition du régime alimentaire des poissons-chats capturés mensuellement. Les tableaux 4 et 5 montrent que la consommation en hiver est nettement plus faible et moins variée qu'en été. L'automne et le printemps reflètent des situations intermédiaires.

Durant l'hiver, le régime alimentaire se compose principalement de zoobenthos (larves de Chironomides et Oligochètes) qui est alors accessible. Il y a en effet à cette époque une forte densité de petites larves de Chironomides (G. Torres, com. pers.) mais les quantités ingérées sont peu élevées, car la basse température de l'eau limite l'activité des poissons-chats. De même, la consommation de zooplancton est 


\begin{tabular}{|c|c|c|c|c|c|c|c|c|c|c|c|}
\hline Mois de l'année & Sep. & oct. & Nov & Dec & Jan & Fev & Mar. & . Mai & Jun . & Jut. & . Aou \\
\hline Effectif de l'échantillon & 13 & 12 & 7 & 39 & 35 & 33 & 22 & 24 & 45 & 27 & 19 \\
\hline Diptères & 92,3 & 91,6 & 71,4 & 69,2 & 45,7 & 57,5 & 50,0 & 95,8 & 86,6 & 85,1 & 100,0 \\
\hline Larves de Chironomes & 92,3 & 91,6 & 71,4 & 69,2 & 45,7 & 57,5 & 50,0 & 95,8 & 86,6 & 81,4 & 100,0 \\
\hline Nymphes de Chironomes & 84,6 & - & - & - & - & - & - & 95,8 & 48,8 & 74,0 & 58,4 \\
\hline Autres Diptères & 7,6 & 8,3 & 14,2 & 2,5 & 2,8 & - & 4,5 & 20,8 & 4,4 & - & - \\
\hline Zooplancton & 53,8 & 50,0 & 42,8 & 35,8 & 20,0 & 24,2 & 63,6 & 100,0 & 46,6 & $.70,3$ & 94,7 \\
\hline Copépodes & 15,3 & 8,3 & - & 30,7 & 17,1 & 21,2 & 50,0 & 100,0 & 42,2 & 62,9 & 89,4 \\
\hline Cladocères & 46,1 & 50,0 & 42,8 & 17,9 & 14,2 & 15,1 & 45,4 & 16,6 & 13,3 & 18,5 & 63,1 \\
\hline Ostracodes & 7,6 & 8,3 & - & 5,1 & 8,5 & 6,0 & - & 8,3 & 6,6 & - & 10,5 \\
\hline Rotifêres & - & - & - & 2,5 & 2,8 & - & - & - & - & - & - \\
\hline 01 igochètes & 69,2 & 75,0 & 71,4 & 35,8 & 25,7 & 15,1 & 36,3 & 16,6 & 46,6 & 70,3 & 10,5 \\
\hline Poissons & 7,6 & 16,6 & - & 15,3 & 2,8 & 6,0 & - & 4,1 & 48,8 & 11,1 & - \\
\hline Gardons & 7,6 & 16,6 & - & 7,6 & - & - & - & 4,1 & 37,7 & 7,4 & - \\
\hline Autres & - & - & - & 7,6 & 2,8 & 6,0 & - & - & 11,1 & 3,7 & - \\
\hline Ecrevisses & - & - & - & - & - & - & - & 4,1 & 26,6 & 3,7 & - \\
\hline Divers & - & 16,6 & 14,2 & 2,5 & 14,2 & 6,0 & 4,5 & 4,1 & 33,3 & 18,5 & - \\
\hline Algues Fil. et Diat. & - & - & 14,2 & 2,5 & 14,2 & 3,0 & - & - & 2,2 & - & - \\
\hline Coléoptères & - & - & - & - & - & - & - & - & 6,6 & - & - \\
\hline Hēmiptères & - & - & - & - & - & - & - & - & 8,8 & 7,4 & - \\
\hline Mollusques & - & - & - & - & - & - & - & - & 6,6 & 3,7 & - \\
\hline Aselles & - & 16,6 & - & - & - & - & - & - & 11,1 & - & - \\
\hline Frai & - & - & - & - & - & - & - & - & 8,8 & 3,7 & - \\
\hline Débris végétaux & - & - & - & - & - & - & - & - & 11,1 & 3,7 & - \\
\hline $\begin{array}{l}\text { Terrestre (araignées, four- } \\
\text { mis, asticots,graines...) }\end{array}$ & - & 8,3 & - & - & - & 3,0 & 4,5 & 4,1 & 11,1 & - & - \\
\hline
\end{tabular}

TableaU IV. - Variations saisonnières de la composition du régime alimentaire des poissons-chats (méthode de l'occurence).

faible. La pression exercée sur les autres espèces de poissons par les poissons-chats les plus âgés est assez forte. C'est à cette époque que des Rotifères, des algues filamenteuses et des Diatomées figurent dans les aliments.

Avec le réchauffement de l'eau, les quantités consommées augmentent en même temps que les disponibilités du milieu. Elles atteignent leur maximum à la fin du printemps.

La consommation de Copépodes est maximale en été, période où la densité de ces organismes est la plus élevée (Lescher-Moutoué, à parâ̂tre). La consommation de nymphes de Chironomides commence 


\begin{tabular}{|c|c|c|c|c|c|c|c|c|c|c|c|}
\hline \multirow{2}{*}{$\begin{array}{l}\text { Mois de l'année } \\
\text { Effectif de l'échantillon }\end{array}$} & \multicolumn{2}{|c|}{ - Sep. Oct . } & - Nov. & \multicolumn{2}{|c|}{ Dec. Jan. } & Fev. & \multicolumn{3}{|c|}{ Mar. Mai. Jun } & \multicolumn{2}{|c|}{ Ju1. Aou } \\
\hline & 13 & 12 & 7 & 39 & 35 & 33 & 22 & 24 & 45 & 27 & 19 \\
\hline Diptères & 130 & 103,4 & 186,5 & 18,4 & 6,8 & 6,6 & 8,1 & 152,9 & 55,2 & 89,3 & 31,8 \\
\hline Larves de Chironomes & 128,2 & 103,3 & 186,4 & 18,3 & 6,6 & 6,6 & 8,0 & 105,5 & 52,2 & 75,6 & 29,0 \\
\hline Nymphes de Chironomes & 1,8 & - & - & - & - & - & - & 47,0 & 2,8 & 13,7 & 2,8 \\
\hline Autres Diptères & 0,07 & 0,08 & 0,14 & 0,02 & 0,17 & - & 0,09 & 0,41 & 0,17 & - & - \\
\hline Zooplancton & 10,0 & 12,1 & 10,0 & 7,8 & 3,5 & 2,2 & 15,9 & 180,0 & 40,7 & 83,1 & 326,3 \\
\hline Copépodes & 1,7 & 0,83 & - & 5,3 & 2,3 & 1,18 & 8,5 & 176,4 & 25,8 & 80,7 & 210,5 \\
\hline Cladocères & 8,0 & 10,8 & 10,0 & 2,15 & 0,76 & 0,93 & 7,4 & 2,4 & 14,2 & 2,4 & 114,6 \\
\hline Ostracodes & 0,15 & $.0,41$ & - & 0,25 & 0,37 & 0,12 & - & 0,8 & 0,06 & - & 1,05 \\
\hline Rotifëres & - & - & - & 0,05 & 0,08 & 0,08 & - & - & - & - & - \\
\hline 01 igochètes & 10,7 & 12,5 & 9,2 & 4,9 & 0,85 & 2,06 & 3,0 & 12,5 & 20,5 & 20,7 & 3,9 \\
\hline Poissons & 0,07 & 0,16 & - & 0,15 & 0,08 & 0,06 & - & 0,04 & 0,48 & 0,11 & - \\
\hline Gardons & 0,07 & 0,16 & - & 0,07 & 0,02 & - & - & 0,04 & 0,37 & 0,07 & - \\
\hline Autres & - & - & - & 0,07 & 0,05 & 0,06 & - & - & 0,11 & 0,03 & - \\
\hline Ecrevisses & - & - & - & - & - & - & - & 0,04 & 0,33 & 0,03 & - \\
\hline Divers & & & & & & & & & & & \\
\hline Co] éoptères & - & - & - & - & - & - & - & - & 0,11 & - & - \\
\hline Hëmiptères & - & - & - & - & - & - & - & - & 0,15 & 0,07 & - \\
\hline Mollusques & - & - & - & - & - & - & - & - & 0,26 & 0,03 & - \\
\hline Aselles & - & 0,16 & - & - & - & - & - & - & 1,13 & - & - \\
\hline
\end{tabular}

Tableau V. - Variations saisonnières de la composition du régime alimentaire des poissons-chats (méthode du dénombrement).

rapidement dès le début de l'envol. L'intensité de cette prédation reflète bien le cycle des émergences qui se fait à Créteil avec deux maximums, le premier et le plus marqué, à la fin du printemps vers :ivril-mai et le second moins important, en août-septembre (G. Torres, com. pers.).

Pendant la période estivale, c'est le déficit en oxygène de la zone limnétique qui perturbe le comportement alimentaire des poissonschats. Ils sont contraints de remonter dans la zone littorale et c'est à ce moment-là que leur régime est le plus diversifié : ils s'accomodent alors des proies qu'ils peuvent trouver sur les berges. La présence de débris végétaux n'est alors qu'accidentelle : ils seraient avalés par hasard en même temps que d'autres aliments.

La consommation d'écrevisses est un phénomène très ponctuel. Cette prédation est maximale au mois de juin, période où les mues 
sont nombreuses (Jestin 1979) et les écrevisses très vulnérables. Parallèlement, l'anoxie du fond, en confinant les poissons-chats et les écrevisses dans la même zone ne peut que favoriser la prédation. C'est ce même phénomène qui explique l'augmentation de la prédation sur les autres poissons. L'importante consommation de Gardons au mois de juin coïncide toutefois avec une très forte mortalité d'individus ligulés à cette époque, sans doute à cause de la température élevée de l'eau. Aussi cette consommation de Gardons peut-elle ne porter que sur des individus déjà morts.

La destruction des pontes des autres espèces n'est pas importante, mais les conditions climatiques ont été très bonnes pendant la période de fraie, de sorte que les éclosions ont été très rapides et que cette source de nourriture a été trop ponctuelle pour être largement consommée. En outre, à cette époque (mai-juin), les poissons-chats sont encore au fond du lac, alors que les autres poissons frayent sur les berges.

\section{3. - DISCUSSION ET CONCLUSIONS}

Il convient de rester critique quant aux résultats obtenus, car la méthode d'analyse utilisée, qui a le mérite de la simplicité, présente des incertitudes. La vitesse de transit stomacal des différentes catégories d'aliments est en effet inconnue, ainsi que les rythmes journaliers d'alimentation. L'interaction de ces deux facteurs avec l'heure d'échantillonnage influence certainement les résultats, surtout en période chaude. L'analyse porte sur l'ensemble des contenus stomacaux et intestinaux, dans le but de perdre moins d'informations, mais elle ne permet donc pas une étude très fine des rations consommées.

La variabilité des possibilités d'identification des aliments introduit en outre des biais systématiques. Les Oligochètes sont probablement sous-estimés, leurs soies étant difficilement visibles à la loupe binoculaire. A l'opposé, les proies digérées lentement sont surestimées.

La méthode de l'occurence donne parallèlement une image déformée en favorisant les petits organismes, souvent plus nombreux, et le dénombrement ne permet pas une bonne comparaison des différentes catégories d'aliments.

L'état des aliments rencontrés rendrait toutefois bien imprécise et délicate l'application de méthodes fondées sur des estimations de poids secs des différentes catégories d'organismes comme cela se pratique chez les Salmonidés (Elliott 1973; Neveu et Thibault 1978). De même, des estimations de volumes relatifs auraient été difficiles à 
obtenir et, de toute façon, elles n'apportent pas d'informations plus intéressantes en l'absence de conversions en unités énergétiques.

L'interprétation des résultats est elle aussi délicate car leur signification dépend de la représentation des échantillons étudiés. A titre d'exemple, l'importante consommation de zooplancton qui se produit au mois d'août, traduit bien la forte densité de Daphnies à cette époque (J.-Y. Bertrand, com. pers.). Ces valeurs sont cependant très surestimées car l'analyse porte sur un échantillon composé principalement de jeunes individus à tendances planctonophages.

La présentation de cette étude rend difficile d'apprécier l'influence de la taille des poissons dans l'analyse des variations saisonnières de la composition du régime alimentaire et, à l'inverse, l'étude par classe de tailles néglige les dates de capture des individus. Seule une approche plus statistique à l'aide d'une analyse factorielle permettrait de décrire la complexité de ces relations trophiques; elle est envisagée dans la poursuite de l'étude au cours d'un second cycle annuel.

Les observations qui précèdent n'en confirment pas moins bien le caractère omnivore et opportuniste du poisson-chat et les changements de comportement alimentaire suivant la taille des individus sont bien nets.

La caractéristique principale qui se dégage de cette étude est la grande adaptabilité de cette espèce aux variations de son milieu. Il semble bien, en effet, que ce soient avant tout les disponibilités et l'accessibilité des ressources offertes qui sont le facteur déterminant du régime. Le poisson-chat a en tout cas une stratégie alimentaire très efficace dans l'exploitation du biotope.

L'espèce n'apparaît pas nuisible aux autres poissons à Créteil car la destruction des pontes n'est pas un caractère majeur de son comportement. La prédation exercée sur les poissons autres que le Gardon ast peu importante en quantité et elle est surtout due aux poissonschats les plus âgés, apparemment peu nombreux. La consommation de Gardons porte sur des individus malades ou déjà morts et ce rôle de "nettoyeur " serait plutôt bénéfique pour l'assainissement du milieu et la limitation du parasitisme (ligulose).

La nuisance du poisson-chat est davantage due au fait qu'il limite par sa présence les ressources alimentaires d'autres espèces pisciaires ayant une valeur esthétique ou économique plus intéressante; or sa grande facilité d'adaptation lui assure un large développement, et cela d'autant qu'aucun prédateur n'intervient pour limiter sa population. 


\section{TRAVAUX CITES}

Bailey (R. M.) et Harrisson (H. M.). 1948. - Food habits of the southern Channel catfish (Ictalurus lacustris, punctatus) in the Des Moines River. Iowa. Trans. Am. Fish. Soc., 75 (1945) : 110-138.

Chesterikoff (A.). 1979. - Etude de la qualité des eaux du lac de Créteil. Rapport Inst. Hydrol. Univ. $P 6: 59 \mathrm{p}$.

Davis (L. T. O.). 1977. - Food habits of the freshwater catfish (Tandanus tandanus, Mitchell) in the Gwydir River, Australia, and effects associated with impoundment of this river by the Copeton Dam. Aust. Jour. of Mar. and Freshwat. Res., 28 : 455-465.

Elliotr (J. M.). 1973. - The food of brown and rainbow trout (Salmo trutta and Salmo gairdneri) in relation to the abundance of drifting invertebrates in a moutain stream. Oecologia (Berl.), 12 : 329-347.

Gerdeaux (D.) et Jestin (J.-M.). 1978. - Exemple d'application du chalut électrifié dans un milieu tempéré très minéralisé. Annls Limnol., 14 : 281-287.

Guiral (D.). 1979. - Etude de la sulfato-réduction dans les sédiments du lac de Créteil. Th. $3^{\text {e }}$ cycle Univ. P 6 (à paraître).

HEARD (R.W.). 1975. - Feeding habits of white catfish (I. catus) from a Georgia estuary. Q. J. Flo. Acad. Sci., 38 (1) : 20-28.

HyNes (H. B. N.). 1950. - The food of freshwater Stiklebacks (Gasterosteus aculeatus and Pygosteus pungitius) with a review of methods used in studies of the food of fishes. J. Anim. Ecol., 19 (1) : 36-58.

JestiN (J. M.). 1979. - Techniques d'échantillonnage, croissance et production de l'écrevisse, Orconectes limosus Raf., du lac de Créteil. Th. Doct. Ing. Univ. P 6: $128 \mathrm{p}$.

Keast (A.) et Welsh (L.). 1968. - Daily feeding periodicities, food uptake rates and dietary changes with hour of the day in some lakes fishes. J. Fish. Res. Bd. Can., 25 : 1133-1144.

KLARBerg (D. P.) et Benson (A.). 1975. - Food habits of Ictalurus nebulosus in acid polluted water of northern west Virginia. Trans. Am. Fish. Soc., 104 : 541-547.

Lescher-Moutoue (F.). - Description des cycles saisonniers de Calanoïda et de Cyclopoïda dans un écosystème lacustre peu profond (Créteil) (à paraître).

Mc CoRmack (J. C.). 1970. - Observations on the food of perch (Perca fluviatilis) in Windermere. J. of Anim. Ecol., 39 (1) : 255-267.

Neveu (A.). 1979. - Les problèmes posés par l'étude de l'alimentation naturelle des populations sauvages de poissons. Bull. Centre Et. Rech. Sci. Biarritz FRA., 12 (3) : 501-512.

Neveu (A.) et Thibault (M.). 1977. - Comportement alimentaire d'une population de truites Fario (Salmo trutta L.) dans un ruisseau des Pyrénées-Atlantiques, le Lissuraga. Annls Hydrobiol., 8 (2) : 111-128.

Repsys (A. J.), Applegate (R. L.) et Hales (D. C.). 1976. - Food and food selectivity of the Black Bullhead, Ictalurus melas, in the lake Poinsett, south Dakota. J. Fish Res. Bd. Can., 33 : 768-775.

Testard (P.). - Le lac de Créteill et son environnement (à paraître).

WALBURG (C. H.). 1975. - Food of young of the year Channel catfish in Lewis and Clark lakes, a Missouri River reservoir. Ann. Midl. Nat., 93 (1) : 218-221. 